\title{
Image Pre-processing techniques comparison: COVID-19 detection through Chest X-Rays via Deep Learning
}

\author{
Arunit Maity ${ }^{1}$, Tusshaar R Nair ${ }^{2}$, Avinash Chandra ${ }^{3}$
}

School of Electronics Engineering, Vellore Institute of Technology, Vellore, Tamil Nadu, India

\section{Article Info}

Volume 7, Issue 6

Page Number: 113-123

Publication Issue :

November-December-2020

\section{Article History}

Accepted : 01 Nov 2020

Published : 10 Nov 2020

\section{ABSTRACT}

The COVID-19 pandemic has had a very devastating effect and has spread rapidly across the world affecting close to 36 million people. Chest radiography is a very important feature which is used for early diagnosis of various diseases. With the increasing pandemic, there is a growing popularity of training Convolutional Neural Networks (CNN) to diagnose and detect COVID-19 from Chest X-Rays. However, publicly available and medically verified datasets for COVID-19 infected chest X-Rays are scarce, which results in the model not generalizing properly. For this purpose, it is important to pre-process and augment the data being used to train the model. Various pre-processing techniques exist like Global Histogram Equalization (GHE), Contrast Limited Adaptive Histogram Equalization (CLAHE) and Top Bottom Hat Transform. In this review, we study and compare all these pre-processing techniques to understand which is the most suitable for developing a CNN model which can classify an image as being infected with COVID-19 or Viral Pneumonia with high efficacy.

Keywords : Radiography, Chest X-Rays (CXRs), COVID-19, Histogram Equalization (HE), Adaptive Histogram Equalization (AHE), Contrast Limited Adaptive Histogram Equalization (CLAHE), Top hat Bottom Hat Transform, image pre-processing, Convolutional Neural Networks (CNNs)

\section{INTRODUCTION}

The COVID-19 pandemic [1][2][3] began in Wuhan, China in December 2019. It didn't take long to spread rapidly across the world. The infection is caused due to the severe acute respiratory syndrome coronavirus 2 (SARS-CoV-2). The typical clinical symptoms of COVID-19 include muscle pain, fever, sore throat, cough, fatigue, headache, shortness of breath or a combination of all of them. The most critical step in helping the fight against the pandemic is timely and effective screening of infected patients who are symptomatic. The most common method for detection of COVID-19 is the reverse transcriptasepolymerase chain reaction (RT-PCR) testing, which is used to detect SARS-CoV-2 RNA from respiratory 
specimens. Although very effective, RT-PCR is very time-consuming, laborious and complicated, while also being short in supply.

Radiology is a very promising field and the information obtained from images is very useful and important in diagnostics and detection. Significant discoveries have been realized by researchers and investigators in imaging studies of COVID-19. In radiology, chest $\mathrm{x}$-rays (CXRs) [4][5][6] play a vital role in providing the information required. Deep Learning [7][8][9] and Convolutional Neural Network $(\mathrm{CNN})$ models have been used earlier for detection of diseases as well as tumours in various organs. There is a growing trend to diagnose more and more diseases using computer aided [10] or automated methods so as to reduce the pressure on radiologists [11]. It is very difficult to have expert and professional radiologists at every hospital, so an automated prediction model can make a huge difference. Artificial Intelligence (AI) based methods are better for rapid COVID diagnosis as they eliminate the reliance on test kits as well as their costs and waiting time like in the case of RT-PCR test [12].

Many radiology images and their datasets are available online, however not all are actually verified by radiologists. Thus, there is a shortage of verified data and not all hospitals have their patients' CXR data uploaded online as a publicly available dataset. Thus, when building a CNN model, it is imperative to pre-process the dataset used to train the model, to ensure the model learns to classify on the basis of pertinent biological features. The pre-processing techniques used in this work are GHE, CLAHE and Top Bottom Hat transform. We feed images using each pre-processing technique to a CNN model to check which technique yields the best results. We also augment the data to ensure the data is generalized and the model doesn't overfit to the training set.
Our CNN model is inspired from VGG-19 and consists of 32 million parameters. We have used transfer learning [13][14] and replaced the head layer of the VGG-19 and added a few more layers with the same design language. We use categorical cross entropy loss and a cyclic learning rate schedule along with Nadam as the optimizer.

Contribution: We found that pre-processing the data being used to train the CNN to detect COVID-19 infection from Chest X-Rays is very important and improves the accuracy. We have thus compared various pre-processing techniques to understand which is the most suitable and precise.

\section{METHODS AND MATERIAL}

\section{A. Dataset}

The dataset used is called the COVID-19 Radiography Database [26]. It consists of a total of 219 images of COVID-19 infected CXRs, 1345 images of viral pneumonia infected patients and 1341 images of normal CXRs. The dataset has been divided into train, test and validation sets and the train/test/validation split ratio we have chosen is an 80:10:10 split. The dataset only comprises posteroanterior (PA) view chest $\mathrm{x}$-rays, since it is necessary that the model is trained consistent data. We resize all the images to $512 \times 512$ to solve the storage constraint and then load the images to the model after implementing image pre-processing techniques on them.

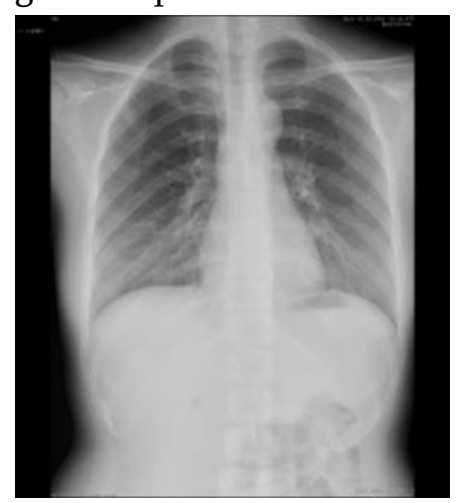

(a) COVID - 19 


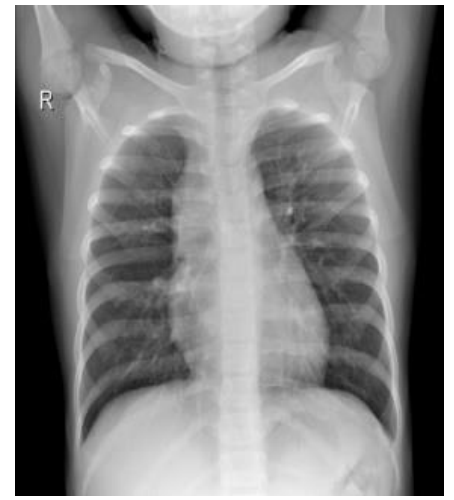

(b) Normal

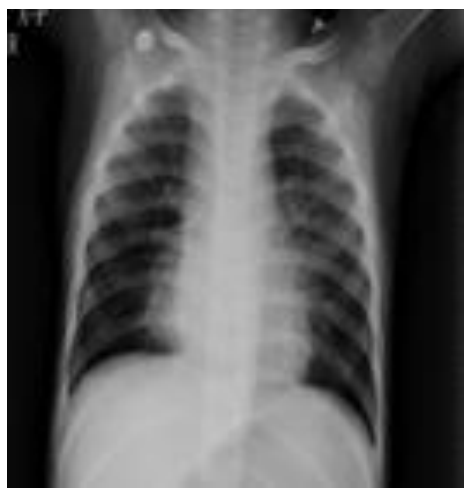

(c) Viral Pneumonia

Fig 1. Chest X-Rays in Data Set

\section{B. Image Pre-processing}

\section{Global Histogram Equalization}

Histogram Equalization [15] is an image processing technique which enhances the quality of the images without losing any information. This method increases the contrast of images by comparing pixels of the image with similar contrast values and distributing their intensities across the histogram.

Histogram Equalization performs the steps of calculating probability mass function and the cumulative distribution function of all the image pixels according to their grey levels, and mapping new grey level values into these pixels after distributing their intensities across the histogram of the image. The histogram represents the number of pixels for each intensity value and the process of Histogram Equalization is done by stretching the intensity range of that image.

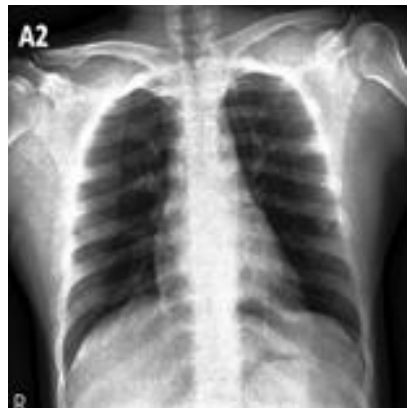

(a) COVID-19

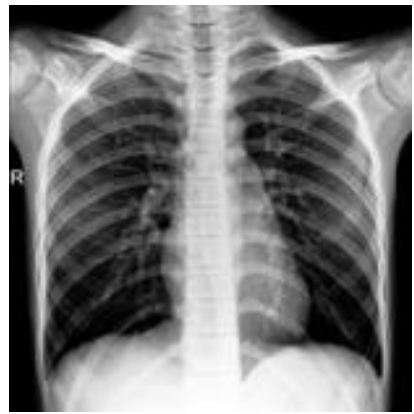

(b) Normal

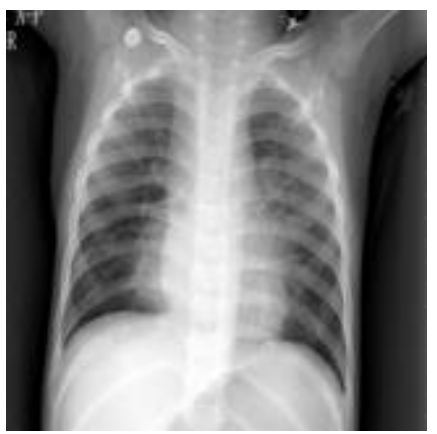

(c) Viral Pneumonia

Fig 2. GHE on Chest X-rays from the dataset

\section{Contrast Limited Adaptive Histogram Equalization}

Adaptive Histogram Equalization [16] tends to overamplify the noise and contrast in the neighbourhood regions of the image since the histogram of tiles in such regions is high. Contrast Limited Adaptive Histogram Equalization (CLAHE) [17][18] is an improved version of adaptive histogram equalization in which the amplification is limited to prevent noise amplification.

In Contrast Limited Adaptive Histogram Equalization (CLAHE), the contrast amplification of pixel is given by the slope of the transformation function. This slope is proportional to the slope of the adjacent 
pixel's cumulative distribution function $(\mathrm{CDF})$ and therefore is also proportional to the value of the histogram to that pixel. CLAHE method limits the amplification of homogeneous regions by clipping the histogram at a particular value before calculating the CDF value of those pixels. This reduces the slope of the CDF. The value at which the histogram is clipped is also known as the clip limit and depends on the normalization of the histogram and also depends on the size of the adjacent region. The part of the histogram that exceeds the clip limit value can be again redistributed equally among all the histogram bins. For implementing CLAHE into our dataset of images, we used a clip limit of 2.0 and a tile grid size of $(8,8)$. Now the significance of tile grid size is that for an image size of 512x512 with tile grid size of $(8,8)$, there would be a total of 4096 tiles for every CXR.
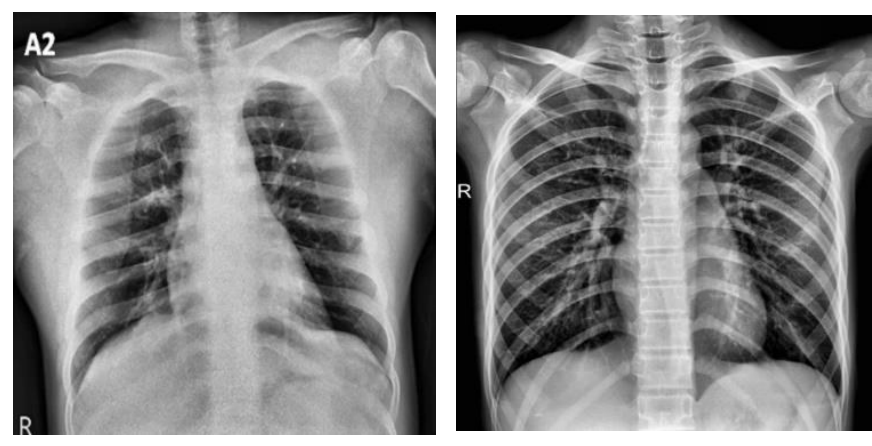

(a) COVID-19 (b) Normal

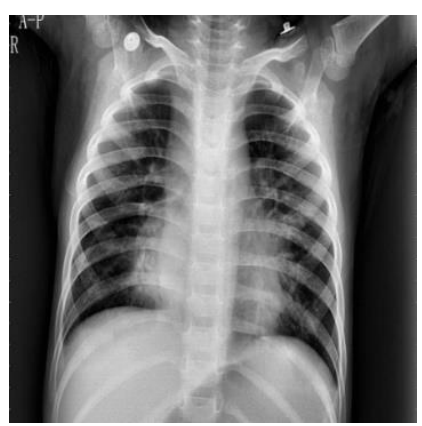

(c) Viral Pneumonia

Fig 3. CLAHE on Chest X-rays from the dataset

\section{Top Bottom Hat Transform (TBH)}

Top-Hat transform is an operation used in Digital Image Processing that extracts small elements and its details from images. There are two types of top-hat transforms: White top-hat transform and Black tophat (Bottom Hat) transform. White top-hat transform is defined as the difference between the input image and its morphological opening. The Bottom Hat transform is defined as the difference between the morphological closing of the image with itself. Tophat transforms are mainly used for feature extraction and image enhancement. The White top-hat transform returns an image, containing the elements of an input image that are smaller than the structuring element, and are brighter than their surroundings. In contrast, the Bottom hat transform returns an image, containing the elements that are smaller than the structuring element, and are darker than their surroundings. The size or width of the elements that are extracted by these transforms can be controlled by choosing the perfect structuring element required.

These transform techniques are mainly used in image segmentation to adjust non-uniform lighting conditions on an image and to provide a better threshold pixel value that is suitable for separating the objects in an image.

Let $\mathrm{A}$ be a grayscale image, mapping points from a Euclidean space. Let B be the chosen structuring element. Then the white top-hat transform of $\mathrm{A}$ is given by:

$\mathrm{WTH}=\mathrm{A}-(\mathrm{A} \circ \mathrm{B})-(1)$

Here, 'o' denotes the morphological opening operation.

The bottom hat transform of $\mathrm{A}$ is given by:

$\mathrm{BTH}=(\mathrm{A} \cdot \mathrm{B})-\mathrm{A}-(2)$

Here, ' ' denotes the morphological closing operation.

The contrast enhanced version of an image is essentially the addition of the image with its White Top Hat transform and their difference with the Bottom Hat transform and is given by:

Aenhanced=A+WTH-BTH -(3)

The image enhancement [19] works because we are indirectly adding the bright regions, which are the result of the white top hat transform and subtracting 
the dark regions which are the results of the bottom hat transform of the original image. For our work, we have taken a structuring element $B$ as a square matrix of ones, with a size of $4 \times 4$.

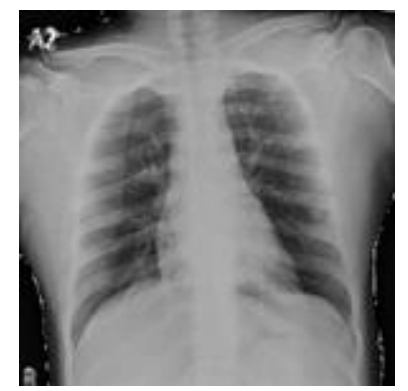

(a) COVID-19

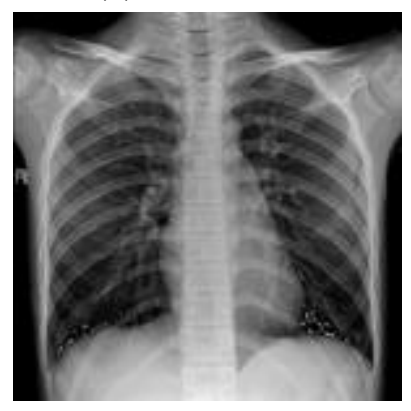

(b) Normal

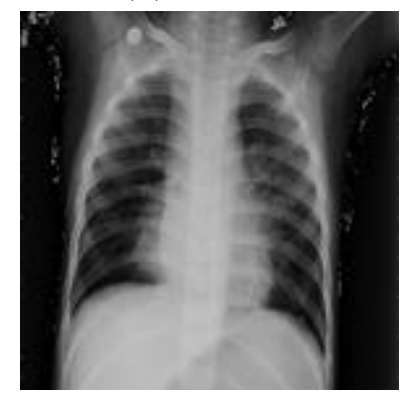

(c) Viral Pneumonia

Fig 4. TBH on Chest X-rays from the dataset

\section{Image Augmentation}

Image augmentation [20][21] is another step after preprocessing the we perform, since it helps in creating more data. This is done to prevent overfitting of the deep CNN model to the limited training data available. Generalization ability of a model is evaluated based on its performance on unseen test images from a different data distribution. We enhance the generalization capability of our model by employing transfer learning and image augmentation. The data augmentation methods that we use in the CXRs are:

\section{Rotation:}

These augmentations are done by randomly rotating the image to the left or right by a certain degree within a defined range. In our case, we require slight rotations so that the lung boundaries and edges do not go out of the image boundary. Hence, we have chosen a rotation range of 0.1 degree.

\section{Width Shift:}

Images are randomly shifted on the horizontal axis by a fraction of the total width within a range to be determined by the users. Here, we need to make sure that the chosen value is such that upon translating the image towards the right or left, the lungs and their edges do not exit the image boundary. We have chosen a fraction value of 0.025 and this corresponds to a range of 12.8 pixels.

\section{Height Shift:}

Images are randomly displaced on the vertical axis by a fraction of the total height within a range to be determined by the users. Again, we need to make sure that the chosen value is such that upon translating the image upwards or downwards, the lungs and their edges do not exit the image boundary. We have chosen a fraction value of 0.025 , corresponding to a range of 12.8 pixels.

\section{Zoom:}

The images are randomly zoomed by a magnitude between 0 and 0.025 . This range was selected to make sure that these augmentations do not make the lungs and their edges exit the image boundary.

\section{Horizontal Flip:}

As the name suggests, the images are randomly flipped horizontally. This was done with the intuition that the model will be able to better diagnose chest radiographs of PA (posteroanterior) as well as AP (anteroposterior) view. 


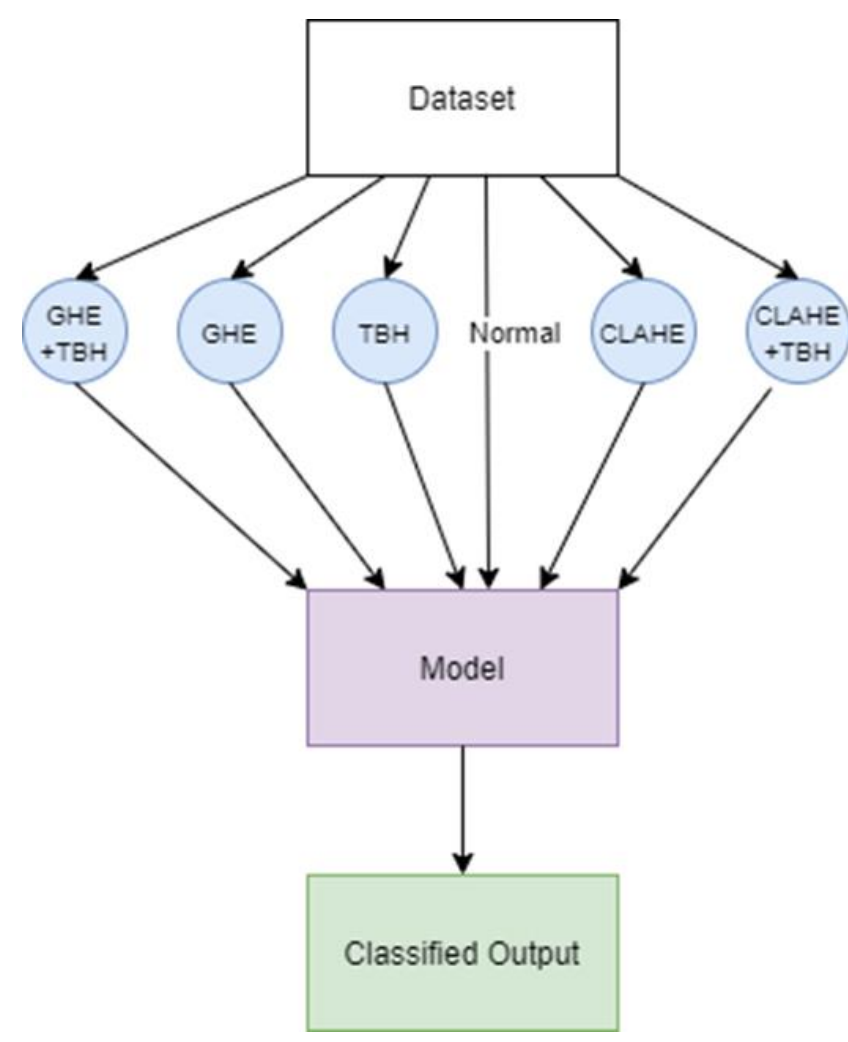

Fig 5. Process flow Diagram

\section{Model Architecture}

Our base model is a pre-existing deep CNN model called VGG-19 [22]. It is a variation of VGG-16 and has close to 32 million parameters. We employ transfer learning [23] by removing the head layer of the VGG-19 and replacing it with a set of custom layers. The head layers we remove include the FC1, FC2 and the SoftMax layers. Our additional layers are mainly composed of Dense, Convolutional, Max Pooling and ReLU layers. The choice of layers was in accordance to the overall architecture design of the VGG-19 model with the intuition that the stability of the neural network will not be compromised. The final model ends with a Soft Max layer which classifies the input into three categories, namely COVID-19, Normal or Viral Pneumonia.

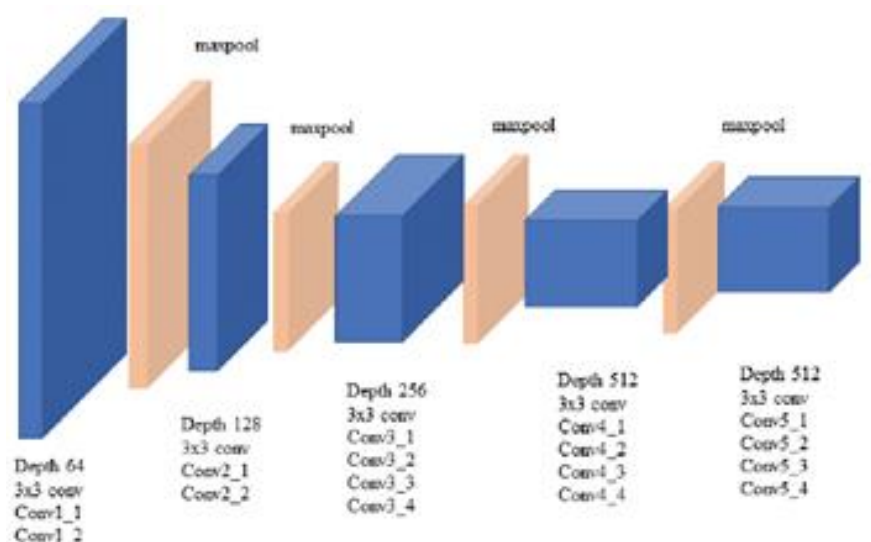

Fig 6. Base VGG-19 Architecture

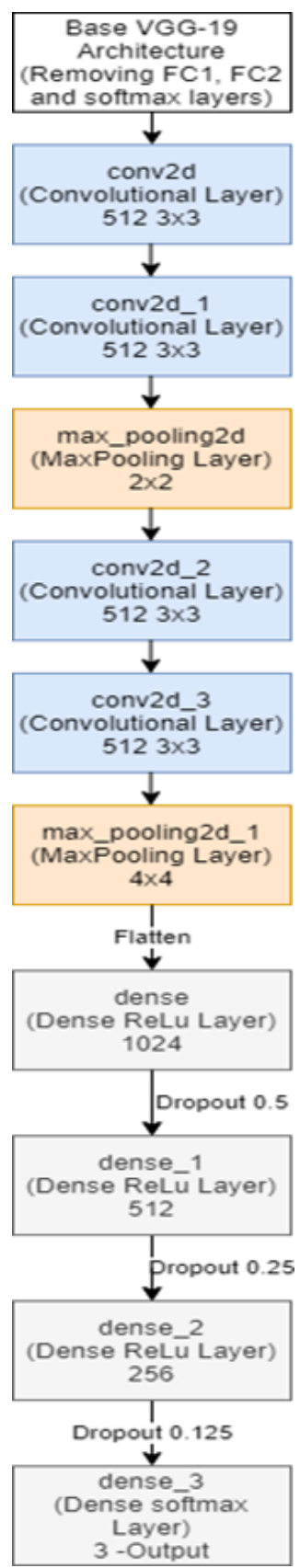

Fig 7. Proposed additional layers to the base model 


\section{E. Implementation Details}

Apart from individually testing out the image preprocessing algorithms, we also test the performance of the model with sequential combinations of the techniques, namely - GHE+TBH and CLAHE+TBH.

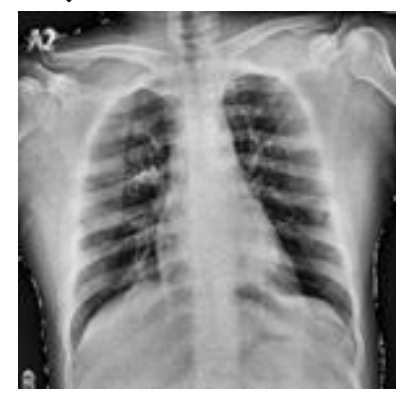

(a) COVID-19

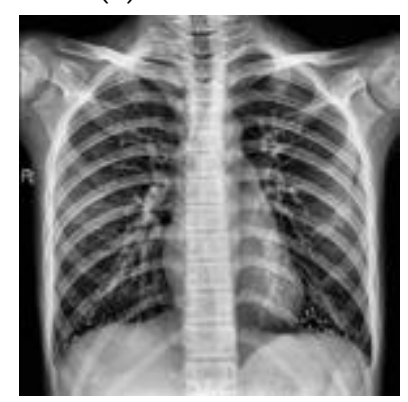

(b) Normal

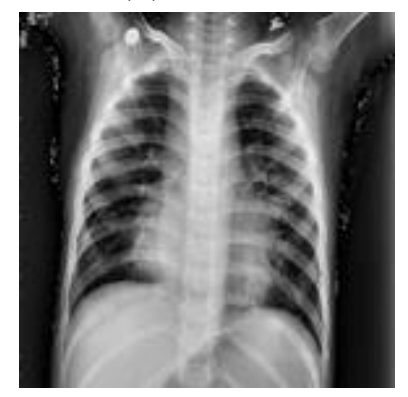

(c) Viral Pneumonia

Fig 8. CLAHE+TBH on Chest X-rays from the dataset

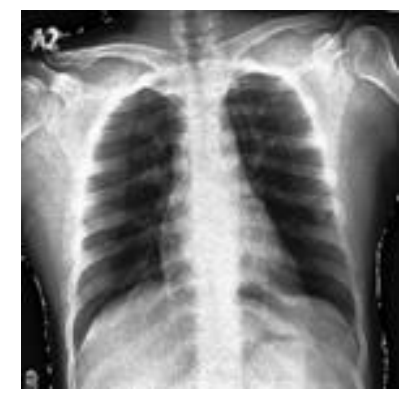

(a) COVID-19

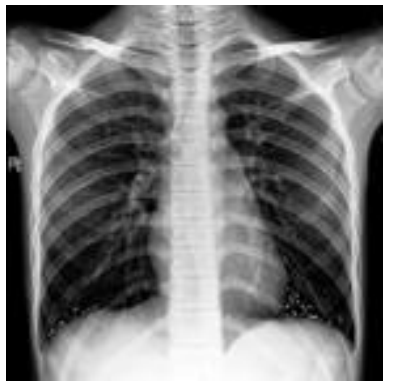

(b) Normal

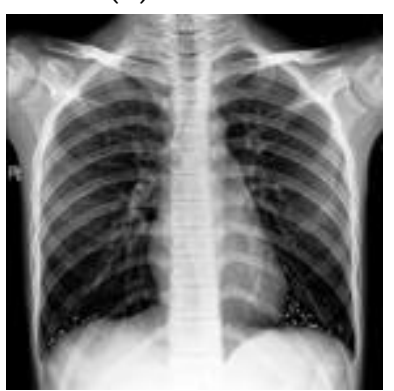

(c) Viral Pneumonia

Fig 9. GHE+TBH on Chest X-rays from the dataset

The specifications of the system used to perform our analysis are as follows:

- GPU - Nvidia Tesla T4 (16 GB)

- No. of Epochs trained - 70

- Training Time - Approx. 3 hours

- TensorFlow version - 2.3.0

- Keras version -2.4 .0$

- OpenCV version - 4.1.2

- Learning Rate Schedule - Cyclic (triangular2 policy)

- Base Learning Rate - 1e-3

- Max Learning Rate - 6e-3

- Loss Function - Categorical Cross Entropy

- Optimizer Function - Nadam

\section{F. Evaluation Metrics}

For the purpose of evaluation of the model, the following metrics [25] were used: precision, recall, F1 score, and accuracy.

1. Precision: Precision is used to evaluate a degree of exactness of the classifiers. It gives a relationship between the true positive predictive values and the fully positive predicted values.

$$
\text { Precision }=\frac{\mathrm{TP}}{\mathrm{TP}+\mathrm{FP}}
$$


2. Recall: Recall is also known as sensitivity which is a measure of the classifier's completeness. It is the ratio between the true positive predicted values and the summation of the true predicted positive values and the predicted false negative values.

$$
\text { Recall }=\frac{\mathrm{TP}}{\mathrm{TP}+\mathrm{FN}}
$$

3. F1-score: F1-score is used to handle the distribution issue with accuracy and is very useful when the dataset comprises of imbalance classes. It is an overall measure of the model's accuracy that combines precision and recall as well.

$$
\mathrm{F} 1-\text { Score }=\frac{2 * \mathrm{TP}}{2 * \mathrm{TP} * \mathrm{FP}+\mathrm{FN}}
$$

4. Accuracy: Accuracy is the most important metric for the results of our classifier model. It is a measure of all the correctly recognized cases.

$$
\text { Accuracy }=\frac{\mathrm{TP}+\mathrm{TN}}{\mathrm{TP}+\mathrm{TN}+\mathrm{FN}+\mathrm{FP}}
$$

\section{RESULTS AND DISCUSSION}

After feeding the datasets after processing them with all the aforementioned image pre-processing techniques and training our proposed CNN model, the results are as follows:

Table 1. Validation Set Accuracy

\begin{tabular}{|l|l|}
\hline $\begin{array}{l}\text { Image Pre-processing } \\
\text { Technique }\end{array}$ & Validation accuracy (\%) \\
\hline Normal & 96.207 \\
\hline CLAHE & 97.586 \\
\hline GHE & 96.207 \\
\hline TBH & 96.207 \\
\hline GHE+TBH & 98.276 \\
\hline CLAHE+TBH & 97.931 \\
\hline
\end{tabular}

From the above, it is evident that the best performance is that of the dataset GHE+TBH, followed by CLAHE+TBH. The validation accuracy describes the accuracy obtained for the validation set, which was created for the purpose of training and simultaneously tuning the hyperparameters of the model. However, the real life performance of the model can only be judged on the basis of the test dataset, since these are chest $\mathrm{x}$-rays which the model has never seen before. The results obtained for the same are as follows:

Table 2. Test data accuracy of the two best image preprocessing techniques

\begin{tabular}{|l|l|}
\hline $\begin{array}{l}\text { Image Pre-processing } \\
\text { Technique }\end{array}$ & Test Accuracy (\%) \\
\hline GHE+TBH & 96 \\
\hline CLAHE+TBH & 94 \\
\hline
\end{tabular}

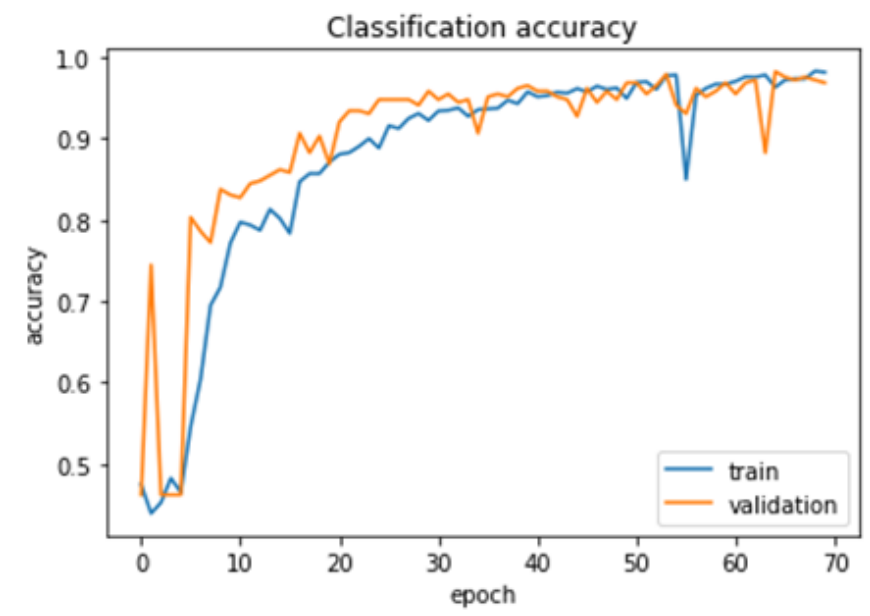

Fig 10. Training Progress of CNN model trained using GHE+TBH processed dataset

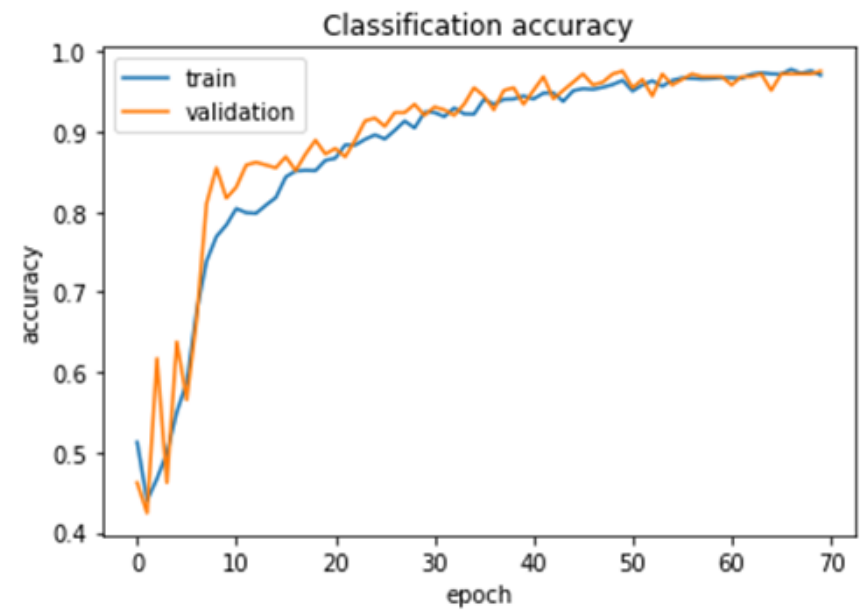

Fig 11. Training Progress of CNN model trained using CLAHE+TBH processed dataset

Now, for further comparison we also compare the evaluation metrics for the CNN models trained on these two image pre-processing techniques. First, we 
observe the metrics for the model trained on the dataset processed through CLAHE+TBH:

Table 3. Evaluation metrics for CLAHE+TBH

\begin{tabular}{|l|l|l|l|}
\hline Infection Type & Precision & Recall & F1-Score \\
\hline COVID-19 & 1.00 & 0.91 & 0.95 \\
\hline Normal & 0.89 & 1.00 & 0.94 \\
\hline $\begin{array}{l}\text { Viral } \\
\text { Pneumonia }\end{array}$ & 0.99 & 0.89 & 0.94 \\
\hline
\end{tabular}

Now, the same metrics for the dataset GHE+TBH are:

Table 4. Evaluation metrics for GHE+TBH

\begin{tabular}{|l|l|l|l|}
\hline Infection Type & Precision & Recall & F1-Score \\
\hline COVID-19 & 1.00 & 0.95 & 0.98 \\
\hline Normal & 0.96 & 0.96 & 0.96 \\
\hline $\begin{array}{l}\text { Viral } \\
\text { Pneumonia }\end{array}$ & 0.95 & 0.96 & 0.96 \\
\hline
\end{tabular}

\section{CONCLUSION}

After analysing the obtained metrics, it is clear that the model performs best when we use an image preprocessing technique. The pre-processing technique we used which gave the best performance was the use of top bottom hat transform followed by global histogram equalization which we abbreviated as GHE+TBH. An added advantage to the model is the precision rate for detecting COVID-19 for the test dataset images which turns out to be 1 . We can also make an argument for the strength of the disease detection power of the model as the precision for pneumonia is also 0.99 which is commendable.

While in no means, is this a production-ready solution, our hope is that since the model's performance in a dataset of CXRs yielded promising results, introducing a public database which consists of multiple sources would help a great deal in improving and further developing the model and making it deployable for rapid and inexpensive testing.

\section{REFERENCES}

[1]. Wu F., Zhao S., Yu B. A new coronavirus associated with human respiratory disease in China. Nature. 2020;579(7798):265-269.

[2]. Huang C., Wang Y. Clinical features of patients infected with 2019 novel coronavirus in Wuhan, China. Lancet. 2020;395(10223):497506.

[3]. World Health Organization. World Health Organization (WHO); 2020. Pneumonia of Unknown Cause-China. Emergencies Preparedness, Response, Disease Outbreak News.

[4]. Rubin, G. D. et al. The role of chest imaging in patient management during the COVID-19 pandemic: A multinational consensus statement from the fleischner society. Radiology (2020).

[5]. Adam J., A. B., Michael C.\& Eber, C. Portable chest X-ray in coronavirus disease-19 (COVID19): A pictorial review. Clin. Imaging (2020).

[6]. Wong, H. et al. Frequency and distribution of chest radiographic findings in COVID-19 positive patients. Radiology (2020).

[7]. Luca B., FrancescoM., AlfonsoR., AntonellaS. Explainable Deep Learning for Pulmonary Disease and Coronavirus COVID-19 Detection from X-rays. Computer Methods and Programs in Biomedicine Volume 196, November 2020, 105608

[8]. Mohammad K. P., Shoaib A. B. SARS n-CoV219 detection from chest $\mathrm{x}$-ray images using deep neural net. International Journal of Pervasive Computing and CommunicationsVolume 16 Issue 5

[9]. Ozturk T, Talo M, Yildirim EA, Baloglu UB, Yildirim O, Rajendra Acharya U. Automated detection of COVID-19 cases using deep neural 
networks with X-ray images. ComputBiol Med. 2020 Jun

[10]. Yu P, Xu H, Zhu Y, Yang C, Sun X, Zhao J (2011) An automatic computer-aided detection scheme for pneumoconiosis on digital chest radiographs. J Digit Imaging 24(3):382-393 11.

[11]. Caobelli F. Artificial intelligence in medical imaging: Game over for radiologists? Eur J Radiol. 2020 May

[12]. Ai, T. et al. Correlation of Chest CT and RTPCR Testing in Coronavirus Disease 2019 (COVID-19) in China: A Report of 1014 Cases. Radiology 200642 (2020)

[13]. Pan, S. J. \& Yang, Q. A survey on transfer learning. IEEE Transactions on knowledge data engineering 22, 1345-1359 (2009).

[14]. T. Carvalho, E. R. S. de Rezende, M. T. P. Alves, F. K. C. Balieiro and R. B. Sovat, "Exposing Computer Generated Images by Eye's Region Classification via Transfer Learning of VGG19 CNN," 2017 16th IEEE International Conference on Machine Learning and Applications (ICMLA), Cancun, 2017, pp. 866870.

[15]. W. Zhihong and X. Xiaohong, "Study on Histogram Equalization," 2011 2nd International Symposium on Intelligence Information Processing and Trusted Computing, Hubei, 2011, pp. 177-179

[16]. Stephen M. Pizer, E. Philip A., John D. A., Robert C., Ari G., Trey G., Bart ter H. R., John B. Z., Karel Z.,Adaptive histogram equalization and its variations,Computer Vision, Graphics, and Image Processing,Volume 39, Issue 3,1987,Pages 355-368

[17]. Georgieva V., Kountchev R., Draganov I. (2013) An Adaptive Enhancement of X-Ray Images. In: Kountchev R., Iantovics B. (eds) Advances in Intelligent Analysis of Medical Data and Decision Support Systems. Studies in
Computational Intelligence, vol 473. Springer, Heidelberg

[18]. Reza, A.M. Realization of the Contrast Limited Adaptive Histogram Equalization (CLAHE) for Real-Time Image Enhancement. The Journal of VLSI Signal Processing-Systems for Signal, Image, and Video Technology 38, 35-44 (2004)

[19]. Kushol, Rafsanjany\& Rahman, A. B. M. Ashikur\& Salekin, Md Sirajus\& Raihan, Md. Nishat. (2018). Contrast Enhancement of Medical X-Ray Image Using Morphological Operators with Optimal Structuring Element. https://arxiv.org/pdf/1905.08545.pdf

[20]. I. Sirazitdinov, M. Kholiavchenko, R. Kuleev and B. Ibragimov, "Data Augmentation for Chest Pathologies Classification," 2019 IEEE 16th International Symposium on Biomedical Imaging (ISBI 2019), Venice, Italy, 2019, pp. 1216-1219

[21]. L. Perez and J. Wang. The effectiveness of data augmentation in image classification using deep learning. arXiv preprint arXiv:1712.04621, 2017

[22]. Karen S., Andrew Z. Very Deep Convolutional Networks for Large-Scale Image RecognitionarXiv:1409.1556

[23]. Tammina, Srikanth. (2019). Transfer learning using VGG-16 with Deep Convolutional Neural Network for Classifying Images. International Journal of Scientific and Research Publications (IJSRP).

[24]. L. Wen, L. Gao and X. Li, "A New Snapshot Ensemble Convolutional Neural Network for Fault Diagnosis," in IEEE Access, vol. 7, pp. 32037-32047, 2019

[25]. Hossin M., Sulaiman M. N., A review on evaluation metrics for data classification evaluationsInt. J. Data Min. Knowl. Manage. Process, 5 (2) (2015), p. 1

[26]. M.E.H. Chowdhury, T. Rahman, A. Khandakar, R. Mazhar, M.A. Kadir, Z.B. Mahbub, K.R. Islam, M.S. Khan, A. Iqbal, N. Al-Emadi, M.B.I. 
Reaz, M. T. Islam, "Can AI help in screening Viral and COVID-19 pneumonia?" IEEE Access, Vol. 8, 2020, pp. 132665 - 132676

\section{Cite this article as :}

Arunit Maity, Tusshaar R Nair, Avinash Chandra, "Image Pre-processing techniques comparison: COVID-19 detection through Chest X-Rays via Deep Learning ", International Journal of Scientific Research in Science and Technology (IJSRST), Online ISSN : 2395-602X, Print ISSN : 2395-6011, Volume 7 Issue 6, pp. 113-123, November-December 2020. Available at doi $\quad$ : https://doi.org/10.32628/IJSRST207614 Journal URL : http://ijsrst.com/IJSRST207614 